\title{
Effects on Ankle Dorsiflexor Activity to Active and Passive Perturbation Condition in Patients With Stroke
}

\author{
Ji-hyun Yuk ${ }^{1}$, BHSc, PT, Jong-duk Choi ${ }^{2}, \mathrm{PhD}, \mathrm{PT}$ \\ ${ }^{1}$ Dept. of Physical Therapy, The Health and Sports Graduated School, Daejeon University \\ ${ }^{2}$ Dept. of Physical Therapy, College of Natural Science, Daejeon University
}

\begin{abstract}
The purpose of this study was to investigate the effects of active and passive postural perturbation on ankle dorsiflexor responses in stroke patients. The subjects consisted of 13 stroke patients. Using wireless electromyography, the patients' ankle dorsiflexor muscle responses were measured under the following conditions: active dorsiflexion ( $\mathrm{AD}$ ), active perturbation (AP), and passive perturbation (PP). Tibialis anterior muscle activity increased most significantly during PP of the affected side (118.64 \pm 56.28 ). The most significant increase for the non-affected side was in $\mathrm{AD}$ (72.64 \pm 24.56$)$. Tibialis anterior muscle activity was compared under each condition. The affected side showed significant differences between PP and $\mathrm{AD}$ and between $\mathrm{PP}$ and $\mathrm{AP}(\mathrm{p}<.05)$. The non-affected side showed not significant differences between each condition. The ratios of tibialis anterior muscle activity under $\mathrm{AP}$ to that under $\mathrm{AD}$ were 1.00 on the affected side and .75 on the non-affected side and the difference was not significant $(\mathrm{p}>.05)$. The ratios of tibialis anterior muscle activity under $\mathrm{PP}$ to that under $\mathrm{AD}$ were 3.30 on the affected side and 1.14 on the non-affected side and the difference was significant $(\mathrm{p}<.05)$. Passive perturbation improved tibialis anterior muscle activity on the affected side, and training based on this approach may have the potential to improve the ankle dorsiflexion of people with stroke.
\end{abstract}

Key Words: Ankle dorsiflexion; Electromyography; Perturbation; Stroke.

\section{Introduction}

After a stroke, many patients experience low extremity movement dysfunction due to muscle weakness (Lawrence et al, 2001), and have mobility problems that must be solved (Lamontagne et al, 2007). Ankle dorsiflexor weakness during stroke patients' functional tasks such as gait is associated with reduced ankle dorsiflexion angles during the swing phase (Lehmann et al, 1987). Moreover, ankle dorsiflexion angle decreases have been reported in more than $45 \%$ of stroke patient (Lamontagne et al, 2002). As such, the pathological causes of foot drop appearing in patients with central nervous system (CNS) lesions include ankle dorsiflexor weakness, plantarflexor hyperactivity, and contracture (Perry and Burnfield, 2010). As shown through several pre- vious studies associated with stroke patients' ambulation, the ankle dorsiflexor is the most important factor during foot drop in patients' walking (Kao and Ferris, 2009; Sorensen et al, 2002).

When normal persons were provided with plantar perturbation during gait and walked against the resistance, the muscle activity of their tibialis anterior increased and their ankle dorsiflexion angles increased clinically significantly. Increased ankle dorsiflexor muscle activity will produce advanced toe clearance and reducing the risk of falls. It will be able to improve walking speed (Blanchette et al, 2011; Rose et al, 2010; Richards et al, 1993).

Having studied normal subjects, Blanchette et al (2011) reported that when the subjects walked against the resistance of plantar perturbation for at least five minutes on a treadmill, their tibialis ante-

Corresponding author: Jong-duk Choi choidew@dju.kr 
rior muscle activity increased during the swing phase and this effect remained for a period of time after the perturbation was removed. In addition, according to an experimental study on body weight-supported treadmill training in patients with stroke or spinal cord injury, short-term low $\mathrm{ex}^{-}$ tremity movement modification during gait training against the resistance of perturbation can lead to longer term improvements (Lam et al, 2011; Reisman et al 2010).

A previous study in which functional electrical stimulation was applied as an intervention in relation to ankle dorsiflexor muscle activation showed improvements in the conditions of stroke patients with foot drop, such as increases in their ankle dorsiflexion angles during swing phases, decreases in their knee flexion angles, and decreases in their ankle plantar flexion angle during toe-off phases, as well as improvements in the patients' ability to avoid obstacles thanks to changes in their overall gait patterns (Kesar et al, 2010; van Swigchem et al, 2011). Ferber et al (2002) applied unexpected perturbations during normal persons' walking to study lower limb muscle activation and kinematic changes. Most previous studies examined ankle dorsiflexor muscle activation or kinematic changes after applying perturbation, and many studies have been conducted with normal subjects or those who were at least capable of independent ambulation among patients with stroke. In contrast, it is almost impossible to find studies in which perturbations under different conditions were applied to those who were capable of independent standing among patients with stroke under different conditions and that drew voluntary responses of only the tibialis anterior in a perturbation environment. Therefore, the aim of this study was to determine the effects of active dorsiflexion, as well as active and passive postural perturbation, on ankle dorsiflexor responses in stroke patients. A further aim was to compare the levels of tibialis anterior muscle activation under different conditions in order to provide basic data for constructing new treatment systems and developing technologies for patients with stroke who are not capable of independent walking or for initial stroke rehabilitation.

\section{Methods}

\section{Subjects}

Participants in this study were 13 hemiplegic patients who were diagnosed with stroke among inpatients and outpatients of the Chungnam National University Hospital in Daejeon. Inclusion criteria for the population were as follows; those who showed muscle weakness due to stroke were classified into at least poor grade in the affected side ankle dorsiflexor with the manual muscle test, were capable of independent standing without any assistance, had no cognitive deficit as shown through a Korean version of Mini-Mental Status Examination (MMSE-K) score not lower than 24 points, had no neurological disorders other than stroke, had no history of musculoskeletal operation on either of the two lower limbs, were free of acute or chronic lesions of the lower extremities, understood this study, and gave voluntary consent to participate. The general characteristics of the subjects are shown in Table 1.

\section{Instrumentation}

A Noraxon Telemyo 2400T (Noraxon Inc., Scottsdale, USA), which is a wireless surface elec-

Table 1. General characteristics of subjects $\quad(\mathrm{N}=13)$

\begin{tabular}{cc}
\hline \hline Characteristics & $\mathrm{n}(\%)$ \\
\hline Gender (male/female) & $11(84.6) / 2(15.4)$ \\
Age (year) & $56.9 \pm 16.0^{\mathrm{a}}$ \\
Hight $(\mathrm{cm})$ & $165.0 \pm 8.8$ \\
Weight (kg) & $65.5 \pm 12.0$ \\
Type of stroke & $10(76.9) / 3(23.1)$ \\
(infarction/hemorrhage) & $6(46.2) / 7(53.8)$ \\
Affected side (left/right) & $208.5 \pm 188.4$ \\
Onset duration (day)
\end{tabular}

\footnotetext{
${ }^{a}$ mean \pm standard deviation.
} 
tromyography (EMG), was used to measure the levels of the muscle activity of the participants' tibialis anterior muscles under different perturbation conditions (Figure 1). Prior to electrode attachment, the sites were shaved and skin surfaces were cleaned with alcohol to reduce skin resistance. Surface electrodes $(\mathrm{Ag} / \mathrm{AgCl})$ were fixed to the muscle bellies of tibialis anterior on the affected and non-affected sides (Hermens et al, 2000).

The signals were collected using a sample frequency of $1000 \mathrm{~Hz}$. Band pass filters between 20 and $450 \mathrm{~Hz}$, and notch filters at $60 \mathrm{~Hz}$ were used. The measured data were analyzed using a MyoResearch Master XP 1.07 (Noraxon Inc., Scottsdale, USA). The EMG signals were processed into root mean squares (RMS) before being analyzed.

A 20 inch Rocker Board (Fitter International Inc., Calgary, Canada) that can be adjusted to three tilt angles-10 degrees, 12 degrees, and 15 degrees-was used as a balance board (Figure 2). The tilt angles were limited to 12 degrees to prevent falls.

\section{Normalization procedure}

To measure tibialis anterior muscle activation, EMG signals obtained as the subjects independently maintained a standing posture were calculated as reference voluntary contraction (RVC) after participants underwent a normalization procedure (Bolgla

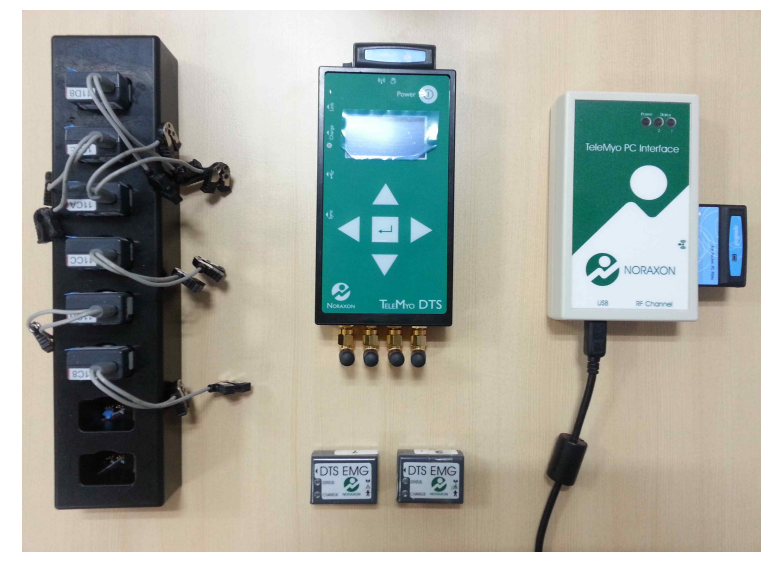

Figure 1. Noraxon Telemyo 2400T. A wireless surface electromyography (EMG) was used to measure the levels of the muscle activity. and Uhl, 2007). The \%RVC of each subject was obtained by recording EMG signals for 5 seconds after a comfortable and stable standing posture was maintained for more than 5 seconds, and the data for the first second and the last second were excluded, such that the data for 3 seconds were used (Kim, 2012).

\section{Muscle activation}

To measure the levels of tibialis anterior muscle activation by condition, voluntary muscle contraction (active dorsiflexion; $\mathrm{AD}$, active perturbation; $\mathrm{AP}$ and passive perturbation; $\mathrm{PP}$ ) were introduced to the participants. To measure $\mathrm{AD}$, each subject sat on a chair at a height at which the subject's feet touched the ground surface and tried maximum possible dorsiflexion. To measure AP, each subject stood in an upright position on a flat surface with the feet spread to shoulder width, while they swayed the trunk posteriorly to the maximum limit at which balance was maintained. To measure PP, the volunteer stood up on a balance board designed to restrain ankle plantar flexion and remained in a static position while posterior perturbation was applied at moments that the subject did not expect it. A method to apply perturbation is that after enough force is applied on hinge which is attached on the back of balance board, hinge is folded and balance board is tilted posteriorly, then the force is applied to passive

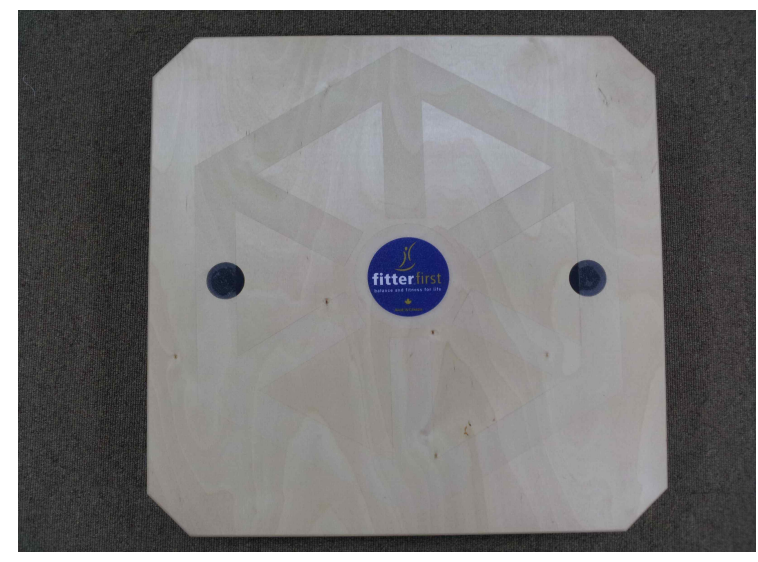

Figure 2. Rocker Board. A 20 inch Rocker Board, which is a balance board, was used to apply to passive perturbation. 
Table 2. Tibialis anterior activation under three different condition

(Unit: \%RVC)

\begin{tabular}{cccccc}
\hline \hline & $\begin{array}{c}\text { Active } \\
\text { dorsiflexion }\end{array}$ & $\begin{array}{c}\text { Active } \\
\text { perturbation }\end{array}$ & $\begin{array}{c}\text { Passive } \\
\text { perturbation }\end{array}$ & F & $\mathrm{p}$ \\
\hline Affected side & $64.45 \pm 37.03^{\mathrm{a}}$ & $54.33 \pm 41.99$ & $118.64 \pm 56.28^{\mathrm{b}, \mathrm{c}}$ & 12.511 & $.001^{*}$ \\
Non-affected side & $72.64 \pm 24.56^{\mathrm{a}}$ & $47.67 \pm 26.74$ & $68.26 \pm 31.51$ & 3.519 & .066 \\
\hline
\end{tabular}

amean \pm standard deviation, ${ }^{b}$ significant difference as compared with active dorsiflexion, ${ }^{c}$ significant difference as compared with active perturbation, ${ }^{*} \mathrm{p}<.05$.

perturbation. This measurement was conducted between parallel bars to prevent falling accidents and the horizontality of the balance board was verified using a level ruler before measurement. Each condition was randomly applied and the level of muscle activation was measured three times. A rest period of 2 minutes was given between each of the conditions (Bolgla and Uhl, 2007) (Figure 3).

\section{Statistical analysis}

In this study, the collected data were analyzed using SPSS Statistics ver. 18. The mean and standard deviation of each general characteristic of the subjects obtained through frequency analysis and $\mathrm{de}^{-}$ scriptive statistics was calculated. Comparisons of the levels of muscle activity by condition between the affected side and the non-affected side were analyzed using one-way repeated analyses of variance. The ratios of tibialis anterior muscle activity under $\mathrm{AP}$ and $\mathrm{PP}$ to that under $\mathrm{AD}$ between the affected side and the non-affected side were analyzed using Wilcoxon signed rank tests. The statistical significance level was set at $a=.05$.

\section{Results}

\section{Tibialis anterior activation}

The levels of tibialis anterior muscle activation by condition on the affected side were measured and the results showed values of $64.45 \pm 37.03$ under $\mathrm{AD}$, $54.33 \pm 41.99$ under $\mathrm{AP}$, and $118.64 \pm 56.28$ under PP. The values were post-hoc tested using least significance differences (LSD) to explain differences between individual conditions. According to the results, tibialis anterior muscle activation showed significant differences between $\mathrm{PP}$ and $\mathrm{AD}$ and between $\mathrm{PP}$ and $\mathrm{AP}(\mathrm{p}<.05)$.

The levels of tibialis anterior muscle activation on the non-affected side were measured by condition
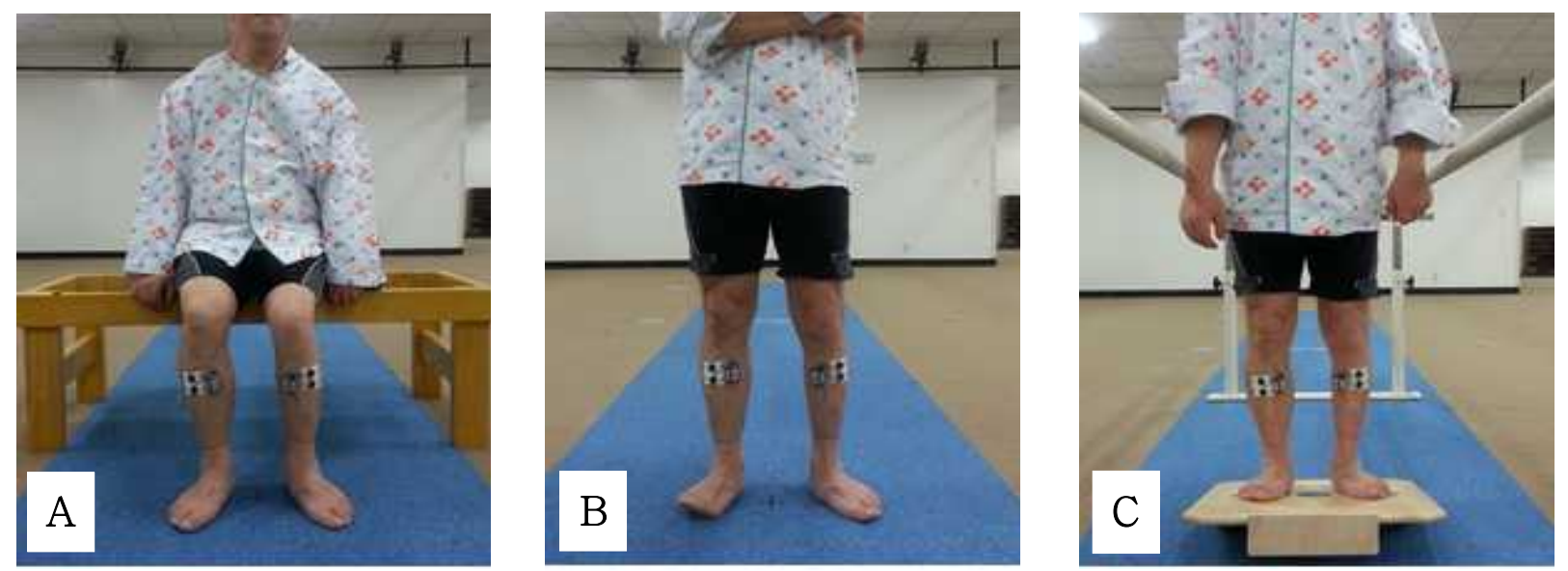

Figure 3. Muscle activation of tibialis anterior (A: active dorsiflexion, $\mathrm{B}$ : active perturbation, C: passive perturbation). To measure the levels of tibialis anterior muscle activation by condition, voluntary muscle contraction (A), active posterior sway (B), and passive posterior perturbation (C) were introduced to the participants. 
Table 3. The ratios of muscle activation under active perturbation and passive perturbation to that under active dorsiflexion

\begin{tabular}{ccccc}
\hline \hline Ratio to active dorsiflexion & Affected side & Non-affected side & $Z$ & $\mathrm{p}$ \\
\hline Active perturbation/Active dorsiflexion & $1.00^{\mathrm{a}}$ & .75 & -1.503 & .113 \\
Passive perturbation/Active dorsiflexion & 3.30 & 1.14 & -2.691 & $.007^{*}$ \\
\hline
\end{tabular}

a percentage of active dorsiflexion value, ${ }^{*} \mathrm{p}<.05$.

and the results showed values of $72.64 \pm 24.56$ under $\mathrm{AD}, 47.67 \pm 26.74$ under $\mathrm{AP}$, and 68.26 \pm 31.51 under PP. The values were post-hoc tested using LSD to ex$^{-}$ plain differences between individual conditions. According to the results, tibialis anterior muscle activation on the non-affected side showed no significant differences between any of the conditions (p>.05) (Table 2).

The ratios of muscle activation under active perturbation and passive perturbation to that under active dorsiflexion

The ratios of tibialis anterior muscle activity under $\mathrm{AP}$ to that under $\mathrm{AD}$ were 1.00 on the affected side and .75 on the non-affected side and the difference was not significant $(\mathrm{p}>.05)$. The ratios of tibialis anterior muscle activity under $\mathrm{PP}$ to that under $\mathrm{AD}$ were 3.30 on the affected side and 1.14 on the non-affected side and the difference was significant $(\mathrm{p}<.05)$ (Table 3).

\section{Discussion}

Stroke is a main cause of severe, long-term disabilities all over the world. Approximately 20\% of stroke survivors cannot adequately control ankle dorsiflexion during walking or show remaining foot drop (Lyons et al., 2002). Foot drop is a result of declined active control of the feet during neurotransmitter changes during walking and it is one of the symptoms of damaged motor functions. Persons with foot drop generally lose the functional ankle dorsiflexion of the affected leg, and thus do not lift the ankles and the toes. As a result, the patients drag the toes of the affected foot on the ground during walking and this becomes a cause of gait disturbance (Kottink et al, 2004; Teasell et al, 2003).

In many previous studies, increases in ankle dorsiflexion angles and improvement in gait speed and balance ability could be found when ankle dorsiflexor muscle activity increased during ambulation (Blanchette et al, 2011; Kesar et al, 2010; Lam et al, 2011; Reisman et al, 2010; Richards et al, 1993; Rose et al, 2010; van Swigchem et al, 2011). In addition, the strength and the coordination of ankle muscles such as tibialis anterior and gastrocneminus are important factors to maintain balance (Kaminski and Hartsell; 2002). In this study, active dorsiflexion, active perturbation, and passive perturbation were applied as methods of increasing stroke patients' ankle dorsiflexion muscle activity in environments other than walking and the levels of tibialis anterior muscle activation on the affected side and the non-affected side were measured.

On the affected side, tibialis anterior muscle activity under PP showed significant increases $(p<.05)$ compared to that under $\mathrm{AD} \quad(\mathrm{p}<.05)$. In addition, when tibialis anterior muscle activity under $\mathrm{AP}$ was compared to that under PP, that under PP showed significant increases $(\mathrm{p}<.05)$. In other words, the affected side showed significant differences between $\mathrm{PP}$ and $\mathrm{AD}$ and between $\mathrm{PP}$ and $\mathrm{AP}(\mathrm{p}<.05) . \mathrm{AD}$ and AP did not show any significant difference. This result may be attributed to participants' decreased balance ability and their fear of falling in AP environments. Whereas previous studies focused on patients capable of walking, this study induced activity in the affected side tibialis anterior muscle in subjects capable of independent standing and affected 
side tibialis anterior muscle activity responses were significant when PP was applied $(\mathrm{p}<.05)$.

This study figures out the ratio of active perturbation (AP) and passive perturbation (PP) on active dorsiflexion $(\mathrm{AD})$. This figure helps to find out how much tibialis anterior activity is increased when perturbation is applied as compared with voluntary muscle contration, and ratio of $\mathrm{PP}$ on $\mathrm{AD}$ is increased more than three times in affected side. As a result, applying perturbation to induce the activity of tibialis anterior is considered as a clinically valuable method.

Movement errors may occur during walking due to several perturbation environments, and motor commands gradually modify muscle activation patterns to adapt to perturbation environments in order to reduce such errors (Kao and Ferris, 2009; Noble and Prentice, 2006; Noel et al, 2009). Fortin et al (2009) provided a variety of walking environments to healthy subjects using elastic bands on a treadmill to study the alteration of lower limb muscle activity patterns. According to the results, 50 or more steps were sufficient to maintain a modified muscle activity pattern; thus, the researchers suggested that movement errors would decrease for 24 hours after initial exposure to a novel environment. In addition, short-term modified low extremity movement patterns obtained in perturbation environments can lead to long-term improvement of the CNS (Lam et al, 2011; Reisman et al, 2010). Through these studies, external stimulations such as perturbations were found to change muscle activity patterns and the altered muscle activity patterns were found to remain for a certain amount of time. In this study, tibialis anterior muscle activity was induced under various conditions and muscle activation changed most significantly during PP on the affected side. This means that in the short term, muscle activity patterns were corrected by perturbation environments provided from the outside. These results are consistent with those of previous studies. However, the non-affected side showed the most significant changes in muscle ac- tivity in $\mathrm{AD}$ environments.

The limitations of this study include the fact that because only 13 patients participated in the study, the sample was not enough to generalize the results and that the results are not sufficient for assessment in relation to the balance and gaits of the stroke patients because the levels of ankle dorsiflexor muscle activation by condition were measured from only one specific muscle-the tibialis anterior-at one point. In future research, this protocol will be applied as an intervention and the balance and gait functions of stroke patients will be evaluated. Furthermore, because there was a big deviation due to not classify the participants' onset duration, a research is necessary to recognize specifically the effect of characteristics on acute, subacute, and chronic periods.

\section{Conclusion}

In this study, active dorsiflexion, active perturbation, and passive perturbation were applied to $13 \mathrm{pa}^{-}$ tients with ankle dorsiflexor weakness due to stroke to identify the conditions in which the patients responded best. This was determined by measuring the activity of the tibialis anterior using EMG. Tibialis anterior activity on the affected side was highest when PP was applied, and tibialis anterior activity on the less affected side was highest when $\mathrm{AD}$ was applied. In addition, when the levels of tibialis anterior muscle activity under individual conditions were compared with each other, tibialis anterior muscle activation on the affected side when PP was applied was highest by a significant margin. The ratios of tibialis anterior muscle activity under $\mathrm{AP}$ to that under $\mathrm{AD}$ were not significantly different between the affected side and the less affected side while the ratios of tibialis anterior muscle activity under PP to that under $\mathrm{AD}$ showed significant differences between the affected side and the less affected side. Therefore, it was found that affected side tibialis anterior muscle activity showed the most meaningful 
responses when PP was applied to patients with ankle dorsiflexor weakness. The results of this study are recommended for application as basic data for constructing new treatment systems and developing technologies for patients with stroke who are not capable of independent ambulation, as well as for initial stroke rehabilitation.

\section{References}

Blanchette A, Lambert S, Richards CL, et al. Walking while resisting a perturbation: Effects on ankle dorsiflexor activation during swing and potential for rehabilitation. Gait posture. 2011; 34(3):358-363.

Bolgla LA, Uhl TL. Reliability of electromyographic normalization methods for evaluating the hip musculature. J Electromyogr Kinesiol. 2007;17(1): 102-111.

Ferber R, Osternig LR, Woollacott MH, et al. Reactive balance adjustments to unexpected perturbations during human walking. Gait posture. 2002;16(3):238-248.

Fortin K, Blanchette A, McFadyen BJ, et al. Effects of walking in a force field for varying durations on aftereffects and on next day performance. Exp Brain Res. 2009;199(2):145-155.

Hermens HJ, Freriks B, Disselhorst-Klug C, et al. Development of recommendations for semg sensors and sensor placement procedures. J Electromyogr Kinesiol. 2000;10(5):361-374.

Kaminski TW, Hartsell HD. Factors contributing to chronic ankle instability: A strength perspective. J Athl Train. 2002;37(4):394-405.

Kao PC, Ferris DP. Motor adaptation during dorsiflexion-assisted walking with a powered orthosis. Gait posture. 2009;29(2):230-236.

Kesar TM, Perumal R, Jancosko A, et al. Novel patterns of functional electrical stimulation have an immediate effect on dorsiflexor muscle function during gait for people poststroke. Phys Ther.
2010;90(1):55-66.

Kim SG. Effect of treadmill gradient training on lower limb muscle activity in chronic stroke patient. J Korea Acad Industr Coop Soc. 2012;13(1):220-226.

Kottink AI, Oostendorp LJ, Buurke JH, et al. The orthotic effect of functional electrical stimulation on the improvement of walking in stroke $\mathrm{pa}^{-}$ tients with a dropped foot: A systematic review. Artif Organs. 2004;28(6):577-586.

Lam T, Pauhl K, Krassioukov A, et al. Using robot-applied resistance to augment body-weight -supported treadmill training in an individual with incomplete spinal cord injury. Phys Ther. 2011;91(1):143-151.

Lamontagne A, Malouin F, Richards CL, et al. Mechanisms of disturbed motor control in ankle weakness during gait after stroke. Gait posture. 2002;15(3):244-255.

Lamontagne A, Stephenson JL, Fung J. Physiological evaluation of gait disturbances post stroke. Clin Neurophysiol. 2007;118(4):717-729.

Lawrence ES, Coshall C, Dundas R, et al. Estimates of the prevalence of acute stroke impairments and disability in a multiethnic population. Stroke. 2001;32(6):1279-1284.

Lehmann JF, Condon SM, Price R, et al. Gait abnormalities in hemiplegia: Their correction by ankle-foot orthoses. Arch Phys Med Rehabil. 1987;68(11):763-771

Lyons GM, Sinkjaer T, Burridge JH, et al. A review of portable FES-based neural orthoses for the correction of drop foot. IEEE Trans Neural Syst Rehabil Eng. 2002;10(4):260-279.

Noble JW, Prentice SD. Adaptation to unilateral change in lower limb mechanical properties during human walking. Exp Brain Res. 2006;169(4): 482-495.

Noel M, Fortin K, Bouyer LJ. Using an electrohydraulic ankle foot orthosis to study modifications in feedforward control during locomotor adaptation to force fields applied in stance. J 
Neuroeng Rehabil. 2009;6:16.

Perry J, Burnfield JM. Gait Analysis: Normal and pathological function. 2nd ed. Thorofare, NJ, Slack Inc., 2010;304-314.

Reisman DS, McLean H, Bastian AJ. Split-belt treadmill training poststroke: A case study. J Neurol Phys Ther. 2010;34(4):202-207.

Richards CL, Malouin F, Dumas F, et al. The relationship of gait speed to clinical measures of function and muscle activations during recovery post-stroke. J Biomech. 1993;26(3):324.

Rose KJ, Burns J, Wheeler DM, et al. Interventions for increasing ankle range of motion in patients with neuromuscular disease. Cochrane Database Syst Rev. 2010;17(2):CD006973.

Sorensen KL, Hollands M, Patla E. The effects of human ankle muscle vibration on posture and balance during adaptive locomotion. Exp Brain Res. 2002;143(1):24-34.

Teasell RW, Bhogal SK, Foley NC, et al. Gait retraining post stroke. Top Stroke Rehabil. 2003; 10(2):34-65.

van Swigchem R, Weerdesteyn V, van Duijnhoven HJ, et al. Near-normal gait pattern with peroneal electrical stimulation as a neuroprosthesis in the chronic phase of stroke: A case report. Arch Phys Med Rehabil. 2011;92(2):320-324.

This article was received October 1, 2013, was reviewed October 1, 2013, and was accepted November 12, 2013. 